\title{
Electron Microscopy and Interface Plasmons Characterization of Cadmium Telluride Thin Film Grown Incommensurately with Weak Bonding on Sapphire
}

\author{
Hesham El-Sherif, Stephen Jovanovic, John Preston and Nabil Basim
}

McMaster University, Hamilton, Ontario, Canada

Cadmium Telluride (CdTe) thin films are important materials for optoelectronic and radiation detection applications. Fabrication of CdTe films has been studied using a variety of substrates via heteroepitaxial growth. As a result of the lattice mismatch strain, the CdTe films usually contain threading and misfit dislocations that limit device performance. Recently, a remote epitaxy technique [1] was demonstrated for homoepitaxial systems by transferring a graphene sheet onto the substrate before deposition of the film. Applied to a heteroepitaxial system, this type of compliant interface would allow for the accommodation of misfit strain due to weakened interfacial bonding. In this current work, a similar compliant interface is created by direct growth [2], without the use of pre-transferred graphene, and instead by the self-assembly of a Tellurium (Te) monolayer at the interface [3]. Utilizing pulsed laser deposition (PLD), high-quality epitaxial CdTe films can be grown on sapphire and then transferred from their substrate to be freestanding leaving the substrate for subsequent growths.

Probe-corrected TEM experiments were used to image the interface in Fig. 1-a and 1-b, show a sharp growth interface where a high-quality CdTe film grows on its (111) orientation on the (0001) sapphire face. The interface in the HAADF-STEM image (Fig. 1-a) shows a monolayer with less on Sapphire with less contrast than the CdTe crystal. A direct electron detector (Gatan's K2 SI) is used to acquire an atomically resolved EELS map of the film (Fig. 1-c) that shows a CdTe(111) with Cadmium up polarity for the crystal, which means a Te termination at the sapphire surface. In addition, the core-loss EELS map (Fig. 1-d) shows a Te-layer nucleating on the sapphire before the CdTe film.

To investigate the bonding at the interface, a monochromated STEM experiment was conducted trying to achieve acceptable both energy and spatial resolution to image and map the Te layer at the interface. Fig. 2-a shows typical atomically resolved STEM-HAADF images acquired with a monochromated beam with around $140 \mathrm{meV}$ energy resolution. With this unique beam, a low-loss EELS map was acquired at the interface region using a US1000FTXP CCD camera at $300 \mathrm{keV}$. The resulted EELS map shows two unique interface intensities around $5.0 \pm 2 \mathrm{eV}$ and $17.5 \pm 2 \mathrm{eV}$. To understand the origin of these interface plasmons we conduced another monochromated experiment at the same imaging and EELS acquiring conditions to study the low-loss plasmons of a pure Te sample (black curve in Fig. 2-b). We demonstrate that these interface plasmons match the characteristic plasmon peaks $(\pi$ and $\sigma+\pi)$ of a pure Te standard with the hexagonal structure that contains weak interchain vdW bonding. This study reveals that unique surface chemistry drives the formation of a stable Te-monolayer termination of sapphire which leads to vdW-like bonding to the heteroepitaxial CdTe film. This allows for a compliant interface, which greatly reduces mismatch defects, as well as the ability for the film to be removed from the substrate and become freestanding. 
a

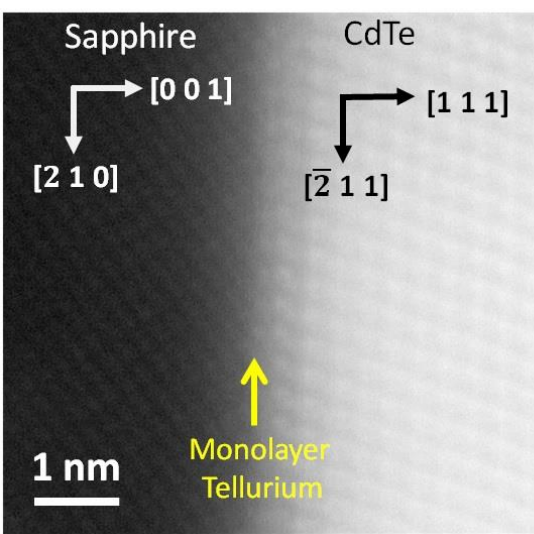

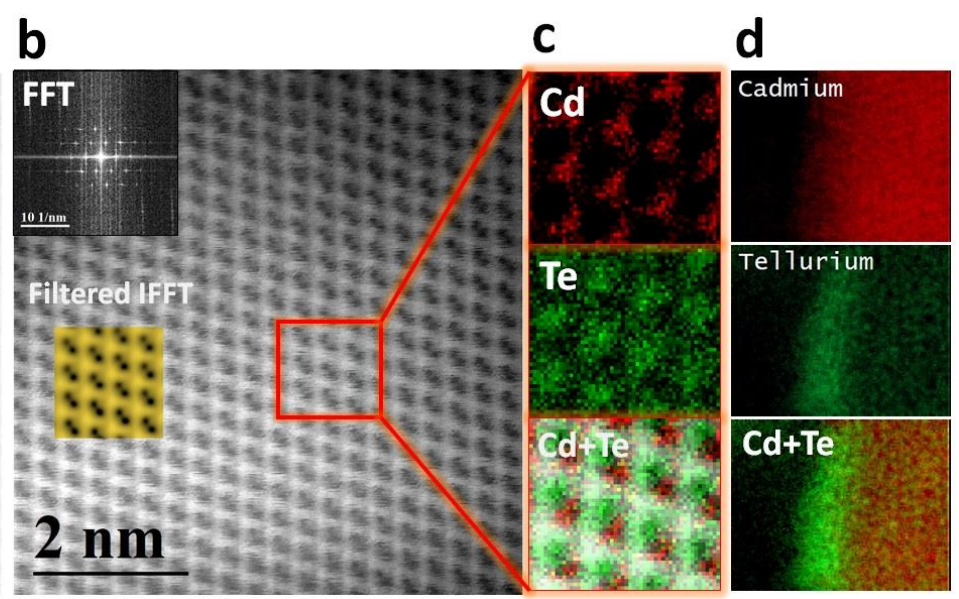

Figure 1. STEM imaging and spectroscopy of the CdTe film. (a) STEM-HAADF image shows that the CdTe grows in the (111) direction and the presence of a monolayer Te at the interface. (b) STEM-ADF image shows the CdTe lattice, its FFT (right-top insert) and a cropped portion of the filtered FFT image (right-middle insert). (c) Atomically resolved EELS maps of Cd and Te shows that the CdTe film starts with Te layer (down) and has a Cd-polarity (up). (d) EELS map at the interface shows a layer of tellurium nucleating on the sapphire before the CdTe film.

a
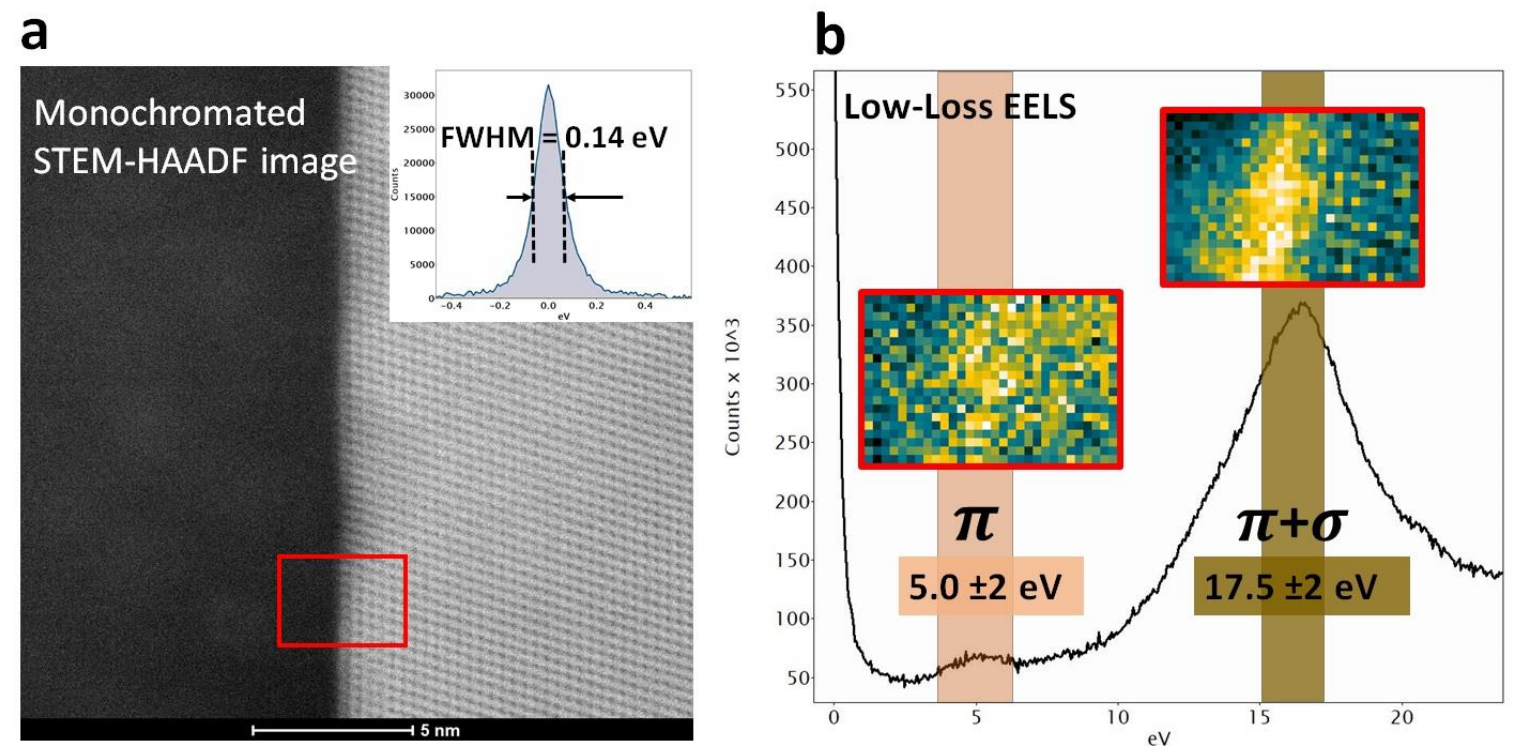

Figure 2. Monochromated STEM experiment for investigating the weak bonding at the interface. (a) STEM-HAADF image achieved with $0.14 \mathrm{eV}$ energy resolution with the possibility of resolving the CdTe lattice fringes and the interface. (b) EELS spectrum (black line) of a pure Tellurium reference shows two main plasmon peaks around $5 \mathrm{ev}$ and $17.5 \mathrm{ev}$. The two inserted maps are the energy-resolved maps acquired at the interface after integrating the spectrum image slices at the corresponding plasmon centers ( \pm 2ev width) found in the Te reference.

\section{References}

[1] Kim, Y., Cruz, S.S., Lee, K., Alawode, B.O., Choi, C., Song, Y., Johnson, J.M., Heidelberger, C., Kong, W., Choi, S. and Qiao, K., 2017. Remote epitaxy through graphene enables two-dimensional material-based layer transfer. Nature, 544(7650), pp.340-343. 
[2] Jovanovic, S.M., Devenyi, G.A., Kuyanov, P., Carvalho, J.L., Meinander, K., LaPierre, R.R. and Preston, J.S., 2018. Epitaxial thin film transfer for flexible devices from reusable substrates. Materials Research Express, 6(2), p.025913.

[3] Hwang, J.Y., Kim, Y.M., Lee, K.H., Ohta, H. and Kim, S.W., 2017. Te monolayer-driven spontaneous van der waals epitaxy of two-dimensional pnictogen chalcogenide film on sapphire. Nano letters, 17(10), pp.6140-6145. 